\title{
Chiral symmetry breaking in lattice QED model with fermion brane
}

\section{Eigo Shintani*}

RIKEN-BNL Research Center, Brookhaven National Laboratory, Upton, NY 11973, USA

E-mail: shintani@riken.jp

\section{Tetsuya Onogi}

Department of Physics, Osaka University, Toyonaka 560-0043, Japan

E-mail: onogi@het.phys.sci.osaka-u.ac.jp

\begin{abstract}
We propose a novel lattice calculation of spontaneous chiral symmetry breaking in QED model with $2+1$ dimensional fermion brane. Considering the relativistic action with gauge symmetry we rigorously carry out path integral in Monte-Carlo simulation with Fermi-velocity relevant to effective coupling constant. We numerically show the evidence of spontaneous chiral symmetry breaking in strong coupling region with chiral condensate, low-lying mode distribution and Nambu-Goldstone boson spectrum in bare Fermi-velocty $v=0.1$. This is a feasible study to investigate the phase structure of Graphene.
\end{abstract}

The 30 International Symposium on Lattice Field Theory - Lattice 2012,

June 24-29, 2012

Cairns, Australia

${ }^{*}$ Speaker. 


\section{Motivation and background}

We consider QED model with $2+1$ dimensional fermion brane $\left(\mathrm{bQED}_{3}\right)$ [1, 2]; in this model fermion has smaller velocity than speed-of-light $c$ whose magnitude has been roughly estimated as $c / 300$ [4]. Tight-binding approximation of Graphene [3] (or similar material formed as honeycomb seat of atoms) has suggested that will possess a particular property which is described by a massless Dirac particle (quasiparticle) [4, 5]. Although there are many analytic studies $[6,7,8,9]$ and numerical works $[10,11]$, it has not been successful to clearly describe the electric property of Graphene.

Many models argue that there should be a signature of spontaneous gap generation due to strong dynamics which indicates the occurrence of semiconductor-insulator transition for suspended monolayer Graphene. However in the experiment there is no evidence of the gap of band structure between electron-hole, and thus it turns out to be permanently conductor. Such inconsistency may be due to the suppression effect of silicon bases, whose dielectric permittivity $\varepsilon$ appears in the denominator of effective coupling, $\alpha_{e}(\varepsilon)=2 \alpha_{e} /(1+\varepsilon) \ll 1$, and thus the impurity of Graphene might be cause of no phase transition. To verify this argument the identification of critical point of effective coupling constant from theoretical study is needed. Our motivation is clarification of phase structure of $\mathrm{bQED}_{3}$ model to understand the transition of Graphene and other kinds of honeycomb crystal.

\section{Brane $\mathrm{QED}_{3}$ model}

As pointed out in [2] the relativistic bQED $_{3}$ model enables us to rigorously deal with path integral under gauge invariant formalism,

$$
S_{\mathrm{bQED}_{3}}=\frac{\beta}{2} \int d t d x^{3}\left(v \vec{E}^{2}+v^{-1} \vec{B}^{2}\right)+\int d t d x^{2} \bar{\psi}\left[i D_{t} \gamma_{t}+i v\left(D_{x} \gamma_{x}+D_{y} \gamma_{y}\right)\right] \psi
$$

with bare Fermi-velocity parameter. In this action we consider not only the Coulomb interactions but also induced magnetic interactions which are $v^{2}$ times weaker than electric field. Since we attempt to treat $\mathrm{bQED}_{3}$ model as local field theory (super-renormalizable), the theoretical parameters, $v$ and $\beta$, are affected by renormalization. The argument of perturbation theory in $\mathrm{bQED}_{3}$ model is that the Fermi-velocity has a logarithmic divergence. The experiment [14] supports the renormalization effect of fermi-velocity of Graphene as suggested in perturbative $\mathrm{bQED}_{3}$ model $[12,13]$ rather than non-relativistic one $[6,7,8,9,10,11]$. The effective coupling constant is defined as the modified form as $\alpha_{e}=1 /(4 \pi \beta v)$ and 2-loop analysis in approximated perturbation explicitly shows the UV fixed point in $\alpha_{e} \gg 1$ [13]. The check of existence of UV fixed point with non-perturbative method is interested in this work.

The gap generation of Graphene is considered to be related with spontaneous chiral symmetry breaking $(\chi \mathrm{SB})$ in $\mathrm{bQED}_{3}$ model. This is analogous to the second order chiral phase transition in $N_{f}=2$ massless $\mathrm{QED}_{3}$ involving the mass gap $[15,16]$. In Eq.(2.1) quasiparticle field $\psi$ has 4 spinor component, $\psi^{t}=\left(\psi_{\sigma}^{A+}, \psi_{\sigma}^{B+}, \psi_{\sigma}^{B-}, \psi_{\sigma}^{A-}\right)$ which corresponds to three kinds of Graphene symmetry; Dirac valleys (degenerating ground energy) $( \pm)$, sublattice symmetry $(A, B)$ and spin of carbon atoms $(\sigma)$. The gamma matrix is given as the tensor structure; $\gamma_{t}=\sigma_{0} \otimes I_{2 \times 2}, \gamma_{i}=$ 
$-i \sigma_{2} \otimes \sigma_{i}(i=x, y, z)$ in which the first part is degree of freedom (DOF) in valley times sublattice and the second one is DOF in spin rotation. Regarding the Graphene symmetry as "flavor" U(4) symmetry in $\mathrm{bQED}_{3}$, whose 16 generators are represented as $\left\{1, \gamma_{5}, i \gamma_{3},\left[\gamma_{3}, \gamma_{5}\right] / 2\right\} \otimes \sigma_{i=t, x, y, z}$ with $\gamma_{5}=i \gamma_{t} \gamma_{x} \gamma_{y} \gamma_{z}$, we define $\gamma_{5}=\operatorname{diag}(1,1,-1,-1)$ "chiral" projection corresponding to left and right chirality (valley chirality) as $U_{L}(2) \times U_{R}(2)$. The chiral condensate $\langle\bar{\psi} \psi\rangle$ is used to be the order parameter of $\gamma_{5} \chi \mathrm{SB}$ as well as QCD.

\section{Lattice calculation of brane $\mathrm{QED}_{3}$ model}

In order to carry out ab initio calculation of $\mathrm{bQED}_{3}$ model, we implement the staggered-type fermion action including the non-compact $\mathrm{U}(1)$ gauge action;

$$
\begin{aligned}
& S_{g}=\sum_{n=(x, y, z, t)}\left[\beta v \sum_{i}\left(\nabla_{4} \theta_{i}(n)-\nabla_{i} \theta_{4}(n)\right)+\beta v^{-1} \sum_{i, j}\left(\nabla_{i} \theta_{j}(n)-\nabla_{j} \theta_{i}(n)\right)\right], \\
& S_{f}=\sum_{m=(x, y, t)}\left[\sum_{i} \eta_{i}(m) \bar{\chi}(m)\left\{U_{i}(m) \chi(m+\hat{i})-U_{i}^{\dagger}(m) \chi(m-\hat{i})\right\}+M \bar{\chi}(m) \chi(m)\right],
\end{aligned}
$$

with differential $\nabla_{i}(m, n)=\delta_{m, n+1}-\delta_{m, n}$, link variable $U_{i}(m)=\exp (i \theta(m))$ and Kawamoto-Smit phase factor $\eta_{i}(m)=\prod_{k=1}^{m_{i-1}}(-1)^{k}$. Conveniently the staggered action in $2+1$ dimension has $U(2) \times$ $U(2)$ (flavor-) chiral symmetry in the continuum limit, and hence we can perform Hybrid-MonteCarlo (HMC) simulation straightforwardly [10, 11, 2].

Here we show the numerical results of chiral condensate, low-lying mode distribution and hadronic spectrum in $N_{s} \times N_{t}=40^{2} \times 20$ and $N_{z}=8$ lattice with fixed $v=0.1$ as a function of fermion mass $M$ and coupling constant $\beta$. For $\mathrm{U}(1)$ field we use periodic boundary for spatial and temporal directions, and for fermion field is periodic boundary for spatial direction and antiperiodic boundary for temporal one. This simulation realizes the lower temperature system thanks to a rescaled temporal size by velocity. To avoid the autocorrelation we use every 20 HMC trajectory per configuration, and the error analysis adopts the Jackknife(JK) method with 10 bin size. Chiral condensate is estimated by 100 noise sources in each configurations.

\section{Chiral symmetry breaking in brane $\mathrm{QED}_{3}$ model}

\subsection{Chiral condensate}

We first show the chiral condensate and chiral susceptibility;

$$
\sigma \equiv\langle\bar{\chi} \chi\rangle=\left\langle\sum_{m} D^{-1}(m, m)\right\rangle, \quad \chi_{m} \equiv \frac{\partial \sigma}{\partial M}=\left\langle\left(\sum_{m} D^{-1}(m, m)\right)^{2}-\frac{1}{V} \sum_{m}\left|D^{-1}(m, 0)\right|^{2}\right\rangle
$$

in several $\beta$ and $M$, where $D^{-1}$ denotes the inverse of staggered fermion matrix. Figure 1 shows $\sigma$ and $\chi_{m}$ have clear dependence of both bare effective coupling $\alpha=1 /(4 \pi \beta v)$ and mass $M . \sigma$ drastically grows up at $\beta v \simeq 0.05-0.06$, and decreasing $M$ this growth is further developing. It seems that the critical point exists around $\beta v \simeq 0.05-0.06$ which corresponds to $\alpha_{c} \simeq 1.3-1.6$, and in $\alpha<\alpha_{c} \sigma$ is close to zero while in $\alpha>\alpha_{c} \sigma$ remains in finite value. The chiral susceptibility is clearly shown to be significant $M$ and $1 /(\beta v)$ dependence similar to critical transition. In $\alpha_{c} \simeq 1.3$ -1.6 there will be a singular point for $\chi_{m}$ in $M=0$ limit, which indicates that $\alpha_{c}$ is expected to be discontinuous at the critical point. 

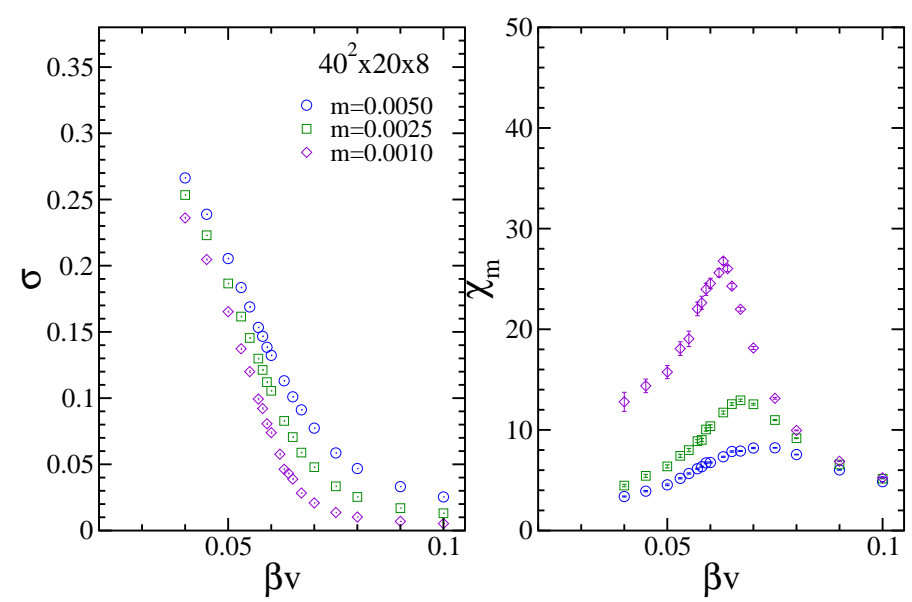

Figure 1: The dependence of the inverse of effective coupling constant $1 /(\beta v)$ for chiral condensate $\sigma$ (left) and chiral susceptibility $\chi_{m}$ (right). Different symbols denote the points in the different fermion masses.

\subsection{Lowlying mode distribution}

Investigation of lowlying mode of massless Dirac operator (Dirac kernel) is helpful to quantify renormalized chiral condensate $\Sigma$ through the Banks-Casher relation;

$$
\Sigma / \pi=\lim _{\varepsilon \rightarrow 0} \lim _{V \rightarrow \infty} \rho(\varepsilon), \quad \rho(\lambda)=\frac{1}{V}\left\langle\sum_{n} \delta\left(\lambda-\lambda_{n}\right)\right\rangle,
$$

in chiral broken phase. Spectral density $\rho(\lambda)$ is as a function of the eigenvalue $\lambda_{n}$ of Dirac kernel (here we consider Dirac kernel in finite volume $V$, and thus distribution of $\lambda$ is a discretized distribution.). The Banks-Casher relation expects that spectral density shows a constant distribution near zero eigenvalue. In finite volume, according to the discussion of random matrix theory (RMT), the "hard edge" [17] of $\rho$ appears at $\lambda=0$ with width $1 /(V \Sigma)$. On the other hand in symmetric phase the spectral density $\rho(\lambda) \sim \lambda^{d-1}$ ( $d$ denotes dimension of fermion), at weak coupling regime. This different behavior is used to not only distinguish the phase in effective coupling constant as well as Figure 1 but also quantitatively estimate the $\Sigma$ in constant region of $\rho$ near $\lambda \sim 0$. In the calculation of lowlying eigenvalue we evaluate 75 different eigenpair (staggered-type Dirac kernel has a pair of eigenvalue with different sign).

In Figure 2 we can clearly see a significant change of the lowlying distribution of $\rho$ above and below $\alpha_{c}$ which is observed in Figure 1. Below $\alpha_{c}(\beta \simeq 0.4)$, there is clear plateau starting from $\lambda=0.002$ in which spectral density is consistent with one in different volume. Near zero point there is also expected "hard edge" whose width becomes narrow increasing volume as in the chiral broken phase. When decreasing the $\alpha$, this "hard edge" disappears and in $\alpha<\alpha_{c} \rho$ becomes monotonically growing. Fitting in $0.002 \leq \lambda \leq 0.007$ with constant function, we obtain $\Sigma$ in each sea fermion mass.

\subsection{Nambu-Goldstone boson spectrum}

In order to confirm $\Sigma$ obtained in spectral density, we attempt to reproduce this from measurement of the spectrum of Nambu-Goldstone (NG) boson particle in bQED 3 . In NG theorem, when 

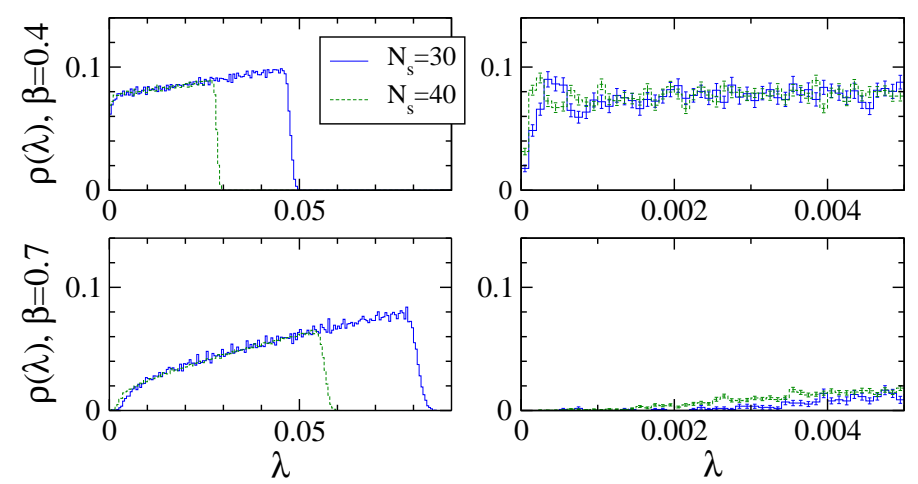

Figure 2: Left panel shows the spectral density at different $\beta$ which are above, close and below $\alpha_{c}$. We also compare different spatial volume. Right panel is a zoomed-up right panel near $\lambda=0$. This is result in sea fermion mass $M_{\text {sea }}=0.001$.

spontaneous $\chi \mathrm{SB}$ occurs, NG bosons appear as an asymptotic one-particle state coupled with axialvector current (or pseudoscalar via Ward-Takahashi identity) operator. If $\alpha>\alpha_{c}$ is in the chiral broken phase, four NG bosons exist as well as pion (and eta) meson in QCD (note that in $\mathrm{bQED}_{3}$ model, since there is no anomaly, flavor singlet particle can be regarded as massless NG boson.) If this picture is true, we can extract the NG boson amplitude and mass from pseudoscalar (PS) correlator in large distance separation between source and sink point. After taking large separation of $x$ direction we expect that PS correlator which is defined as the exact NG boson operator in staggered-type fermion approaches to the following form:

$$
\lim _{x \gg 1} G_{\mathrm{PS}}(x)=\left|Z_{N G}\right|^{2}\left(e^{-m_{\mathrm{NG}} x}+e^{-m_{\mathrm{NG}}\left(N_{s}-x\right)}\right) /\left(2 m_{\mathrm{NG}}\right), \quad f_{\mathrm{NG}}=2 M Z_{\mathrm{PS}} m_{\mathrm{NG}}^{-3 / 2},
$$

where $f_{\mathrm{NG}}$ is a corresponding quantity to pion decay constant in QCD although its mass dimension is $1 / 2$ in $2+1$ dimension. Here we set the source operator in the origin as point source.

Figure 3 shows the clear shape of exponential function of $G_{\mathrm{PS}}$, and effective mass plot explicitly illustrates that in $\alpha>\alpha_{c}$ there is plateau in $x \geq 9$, however in $\alpha<\alpha_{c}$ plateau is not observed. This result indicates that NG boson state following in Eq.(4.3) appears as a consequence of spontaneous $\chi \mathrm{SB}$ in $\alpha>\alpha_{c}$.

Using the value obtained from fitting $G_{\mathrm{PS}}$ with function in Eq.(4.3), we show the lattice results of $f_{\mathrm{NG}}$ and $m_{\mathrm{NG}}^{2} / M$ in each NG boson mass squared in Figure 4. To evaluate the chiral condensate from these results we can use the GMOR relation; $\Sigma=f_{0} m_{\pi}^{2} /(2 M)$ as in the case of QCD where $f_{0}$ is a value of $f_{\mathrm{NG}}$ in $M=0$. According to the loop calculation in chiral perturbation theory (ChPT) in 3-dimension, we set the fitting function

$$
f_{\mathrm{NG}}=f_{0}\left(1+m_{\mathrm{NG}} /\left(4 \pi f_{0}^{2}\right)\right)+c m_{\mathrm{NG}}^{2}, \quad m_{\pi}^{2} / M=2 B\left(1-m_{\mathrm{NG}} /\left(4 \pi f_{0}^{2}\right)\right)+d m_{\mathrm{NG}}^{2},
$$

where $f_{0}, B, c$ and $d$ are fitting variables. We introduce $c$ and $d$ as correction terms to higher order effect than LO ChPT. We attempt to compare the extrapolated result with different fitting range of $m_{\pi}^{2}$, and including the data at $M=0.005$ to extrapolate $M=0$ limit we use a function in Eq.(4.4) besides using data up to $M=0.0025$ we exclude $c$ and $d$ terms. In Figure 4 we see that in $\alpha>\alpha_{c}$ $(\beta=0.4,0.45,0.5)$ the chi-square fitting with both NLO ChPT and NLO CHPT + linear term 

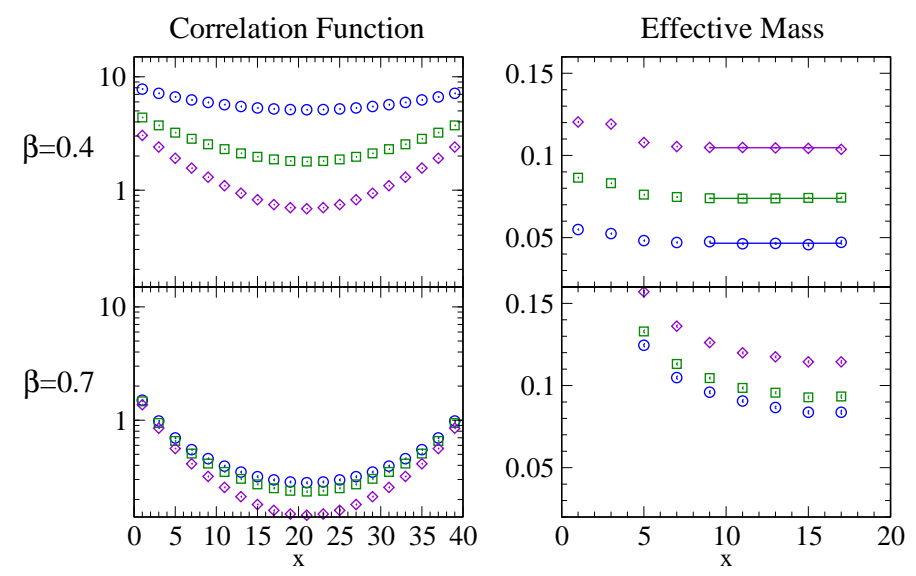

Figure 3: $G_{\mathrm{PS}}(x)$ (left) and effective mass plot (right) at different $\beta$ in $M=0.001$ (circle), $M=$ 0.0025 (square) and $M=0.005$ (diamond). The straight lines are fitted result.

Table 1: Fitting results of chiral condensate obtained by spectral function via Banks-Casher relation and ChPT with NLO and NLO+linear. We also describe the value of $\chi^{2} /$ dof. The error is only statistical one.

\begin{tabular}{cccccc}
\hline \hline$\beta$ & $\Sigma$ (spectral) & $\Sigma($ NLO ChPT $)$ & $\chi^{2} /$ dof & $\Sigma($ NLO ChPT+linear $)$ & $\chi^{2} /$ dof \\
\hline 0.4 & $0.2433(48)$ & $0.2587(8)$ & $12.2(4.1)$ & $0.2657(15)$ & $6.1(2.9)$ \\
0.45 & $0.2140(45)$ & $0.2243(7)$ & $4.1(2.3)$ & $0.2275(13)$ & $2.7(1.9)$ \\
0.5 & $0.1694(51)$ & $0.1807(6)$ & $1.5(1.4)$ & $0.1766(13)$ & $2.9(2.0)$ \\
\hline \hline
\end{tabular}

works better than in $\alpha<\alpha_{c}$. This is also consistent with picture that spontaneous $\chi \mathrm{SB}$ above $\alpha_{c}$ occurs in accompany with NG boson. In table 1 we show preliminary results of comparison of $\Sigma$ with three different ways in above $\alpha_{c}$. These are consistent results within $1-2 \sigma$ under a few $\%$ accuracy.

\section{Summary and discussion}

We perform the ab initio calculation of QED model with $2+1$ dimensional fermion brane $\left(\mathrm{bQED}_{3}\right)$ in Monte-Carlo method. Due to preserving gauge symmetry we take into account not only coupling constant but also fermi-velocity as theoretical parameters. In this proceedings we numerically show the strong evidence of $\chi \mathrm{SB}$ and critical coupling constant from three kinds of way; chiral condensate, spectral density and NG boson spectrum. Although we fix the "bare" velocity parameter in 0.1 which is relatively larger than naive estimate $\left(v \sim O\left(10^{-3}\right)\right)$, we have the consistent result with expected in $\chi \mathrm{SB}$ phenomena. This is feasible study to search the applicability to study of phase structure of Graphene.

The calculations were performed by using the RIKEN Integrated Cluster of Clusters (RICC) facility. This work is supported by the Grant-in-Aid of the Japanese Ministry of Education (No. 20105002, 23105714(MEXT KAKENHI grant)). 

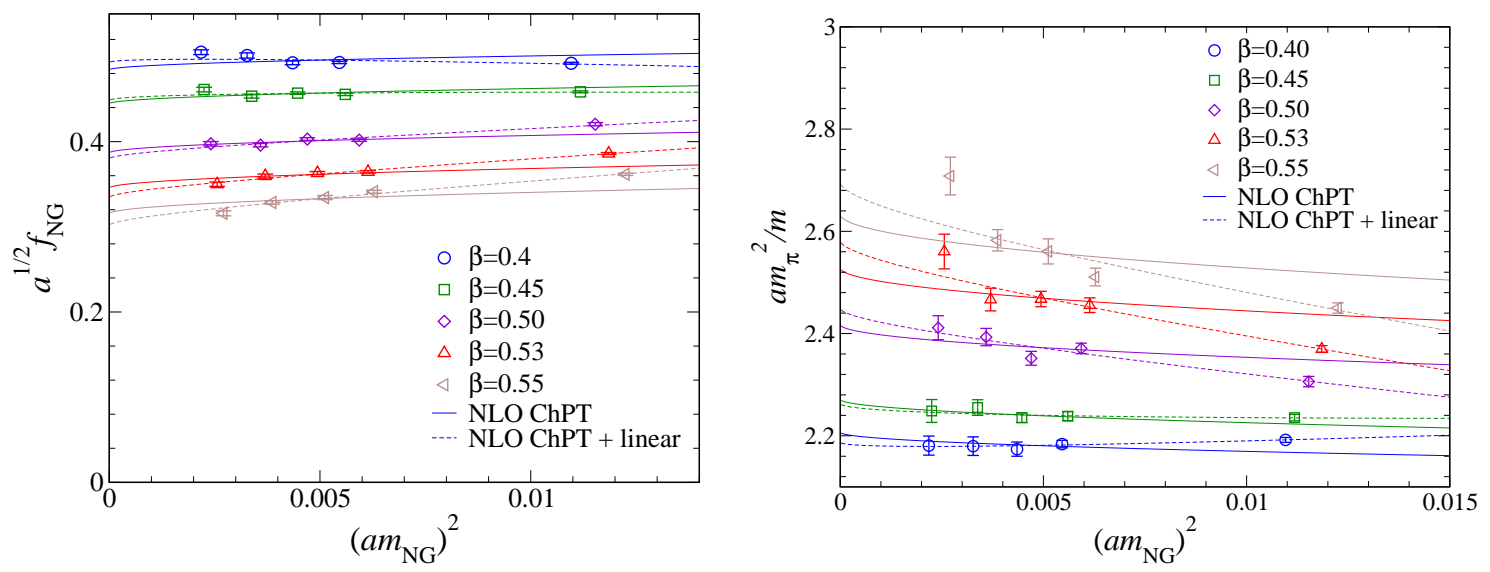

Figure 4: $M$ dependence of $f_{\mathrm{NG}}$ (left) and $m_{\pi}^{2} / M$ (right). Solid line denote the LO ChPT in the range of $0.001 \leq m_{\mathrm{NG}}^{2} \leq 0.0025$ and dashed one denotes the fitting function in LO ChPT plus linear term in the range of $0.001 \leq m_{\mathrm{NG}}^{2} \leq 0.005$.

\section{References}

[1] E. V. Gorbar, V. P. Gusynin and V. A. Miransky, Phys. Rev. D 64, 105028 (2001).

[2] E. Shintani and T. Onogi, arXiv:1203.1091 [hep-lat].

[3] K. S. Novoselov, et al., Science 306, 666 (2004); K. S. Novoselov, et al., Nature 438, 197 (2005); A. K. Geim and K. S. Novoselov, Nature Matter 6, 183 (2007).

[4] P. R. Wallace, Phys. Rev. 71, 622 (1947).

[5] G. W. Semenoff, Phys. Rev. Lett. 53, 2449 (1984).

[6] O. Vafek, Phys. Rev. Lett. 98, 216401 (2007).

[7] D. T. Son, Phys. Rev. B 75, 235423 (2007).

[8] O. V. Gamayun, E. V. Gorbar and V. P. Gusynin, Phys. Rev. B 81, 075429 (2010).

[9] V. Juričić, I. F. Herbut and G. W. Semenoff, Phys. Rev. B 80, 081405(R) (2009).

[10] J. E. Drut and T. A. Lahde, Phys. Rev. B 79, 165425 (2009).

[11] W. Armour, S. Hands, C. Strouthos, Phys. Rev. B 81, 125105 (2010).

[12] J. González, F. Guinea and M. A. H. Vozmediano, Nucl. Phys. B 424, 595 (1994).

[13] V. N. Kotov, et al., arXiv:1012.3484 [cond-mat.str-el].

[14] D. C. Elias, et al., Nature Phys. 7, 701 (2011).

[15] R. D. Pisarski, Phys. Rev. D 29, 2423 (1984).

[16] T. W. Appelquist, M. Bowick, D. Karabali and L. C. Wijewardhana, Phys. Rev. D 33, 3704 (1986).

[17] P. H. Damgaard, U. M. Heller, R. Niclasen and K. Rummukainen, Nucl. Phys. B 583, 347 (2000) [arXiv:hep-lat/0003021]. 